\title{
BMJ Open Prevention of head louse infestation: a randomised, double-blind, cross-over study of a novel concept product, $1 \%$ 1,2-octanediol spray versus placebo
}

\author{
Ian F Burgess, Elizabeth R Brunton, Rebecca French, Nazma A Burgess
}

To cite: Burgess IF, Brunton ER, French R, et al. Prevention of head louse infestation: a randomised, double-blind, cross-over study of a novel concept product, 1\% 1,2-octanediol spray versus placebo. BMJ Open 2014:4:e004634. doi:10.1136/bmjopen-2013004634

- Prepublication history and additional material paper is available. To view please visit the journal (http://dx.doi.org/ 10.1136/bmjopen-2013004634).

Received 6 December 2013 Revised 1 May 2014 Accepted 6 May 2014

CrossMark

Medical Entomology Centre, Insect Research \& Development Limited, Cambridge, UK

Correspondence to Ian F Burgess; ian@insectresearch.com

\section{ABSTRACT}

Objectives: To determine whether regular use of a spray containing 1,2-octanediol $1 \%$, which has been shown to inhibit survival of head lice, is able to work as a preventive against establishment of new infestations.

Setting: Randomised, double-blind, cross-over, community study in Cambridgeshire, UK.

Participants: 63 male and female schoolchildren aged 4-16 years judged to have a high risk of recurrent infestation. Only the youngest member of a household attending school participated.

Interventions: Participants were treated to eliminate lice, randomised between $1 \%$ octanediol or placebo sprays for 6 weeks then crossed-over to the other spray for 6 weeks. Parents applied the sprays at least twice weekly or more frequently if the hair was washed. Investigators monitored weekly for infestation and replenished supplies of spray.

Primary and secondary outcome measures:

The primary endpoint was the time taken until the first infestation event occurred. The secondary measure was safety of the product in regular use.

Results: Intention-to-treat analysis found a total of 32 confirmed infestations in 20 participants, with 9 of them infested while using both products. In these nine participants the time to first infestation showed a significant advantage to $1 \%$ octanediol $(p=0.0129)$. Per-protocol analysis showed only trends because the population included was not large enough to demonstrate significance. There were no serious adverse events and only two adverse events possibly related to treatment, one was a case of transient erythema and another of a rash that resolved after 5 days.

Conclusions: Routine use of $1 \%$ octanediol spray provided a significant level of protection from infestation. It was concluded that this product is effective if applied regularly and thoroughly.

Trial registration number: ISRCTN09524995.

\section{INTRODUCTION}

Head louse infestation continues to be common and widespread despite recent development of treatment products that are

\section{Strengths and limitations of this study}

- As a pragmatic study, the results are an indication of how the product could perform in consumer use.

- The primary limitation of the study was that the risk for infestation of each participant was unknown.

- The results demonstrate the inconsistency with which even motivated people might use the product.

- The study was able to demonstrate a statistically viable outcome for the data.

not affected by resistance to insecticides. There are numerous treatment choices in European countries but, although effective for most users, some children are repeatedly infested. Sometimes this is because caregivers are not successful when using the treatment but often recently treated children are quickly reinfested.

When discussed with concerned parents, apart from effective treatments, most of them wish for a product that can protect children against infestation. Some have interpreted this as using a repellent. ${ }^{1}{ }^{2}$ However, repellents, by their nature, are volatile and therefore not persistent on hair, which means they have limited longevity, especially if the application is not thorough. ${ }^{2}$ Also, because lice crawl from one head to another rather than seeking hosts, the chemicals designed to disrupt the host-seeking function in flying insects may have no activity against crawling lice. In any case, it is recognised that mosquito repellents have limitations of effectiveness so that users may suffer occasional bites. If similar failures occurred with lice, infestations could become established without being noticed.

In the past it was mistakenly believed that insecticides with a residual action could 
protect against reinfestation for several weeks. ${ }^{3}$ This was probably effective for some people but residual effects were inconsistent and systematically leached by hair washing so that the level of insecticide quickly became sublethal for any lice moving onto the hair. ${ }^{3-6}$ Inevitably, lice in contact with low levels of insecticide were selected for resistance to pyrethroid and malathion insecticides in the early 1990s.

The alternative prevention strategy is regular use of a product that prevents lice from establishing an infestation rather than repelling them. This was never true for conventional insecticides, although anecdotes suggest it may have been widely practised, but regular use of low doses of cosmetically acceptable, physically acting chemicals that disrupt the cuticular lipids of lice should kill insects in contact with the treated hair and limit the risk of an infestation establishing. We know that 1,2-octanediol $5 \%$ is effective in eliminating an established head louse infestation. ${ }^{8}$ We also observed during preclinical studies of 1,2-octanediol that $1 \%$ solutions were able to kill lice, albeit more slowly, and inhibit egg laying. This report describes a randomised, doubleblind, cross-over, clinical investigation of a spray containing $1 \%$ 1,2-octanediol, which was developed as a preventive of this type, compared with placebo.

\section{MATERIALS AND METHODS}

\section{Participants}

We recruited participants in a similar way to previous investigations by local radio advertising and by writing to families who had participated in previous clinical trials and expressed a wish to participate in further research. Prospective participants were sent an information booklet and if, after reading, they wished to take part an appointment was made for an appropriate date to start the study.

Unlike other studies, only the youngest member of the family who was attending school was recruited to the study. Thus, the minimum age was 4 years and the maximum 16 years. Other members of the household were not included so they could act as potential sources of infestation for participants.

Everyone joining was conducted through a standardised consent/assent procedure and then assessed for presence of head lice using a plastic head louse detection comb (PDC, KSL Consulting, Denmark). This was mainly to provide information about the person's current risk status because everyone was treated to ensure all participants started free from infestation. Other household members who were infested at this time were offered treatment to reduce the risk of an immediate reinfestation pressure on the index member.

All participants gave baseline data on age, gender and hair characteristics as well as information on current medications and medical history. All treatments and assessments were conducted in participants' homes. There was no payment for participation.
Eligibility and inclusion criteria were being of appropriate age, as described above; being at risk of reinfestation based on previous individual and family history and being willing to participate for the estimated 14 weeks of the study. Exclusion criteria were a history of allergy or sensitivity to components of the test product or placebo; of long-term scalp disorders, such as impetigo or psoriasis; pregnancy or breast feeding and participation in other clinical studies within 1 month prior to entry.

\section{Ethics}

A Clinical Trial Notification was also made to the Medicines and Healthcare Products Regulatory Agency in the UK, reference CI/2012/0032. Parents provided written consent for the participating child. Participants also provided written assent. Each participant's general practitioner was informed of their taking part.

The study was conducted in conformity with the principles of the Declaration of Helsinki and the International Conference on Harmonisation (ICH) Guidelines and European Standard for Good Clinical Practice (GCP).

\section{Study medications}

This was a randomised, double-blind, cross-over study of $1 \%$ 1,2-octanediol in a hair-conditioning base (Hedrin Protect \& Go, Thornton \& Ross Ltd, UK). It was supplied in $100 \mathrm{~mL}$ trigger spray high density polyethylene (HDPE) plastic bottles, used like a leave-in detangler conditioning spray, applied twice weekly to washed and towel-dried hair. More frequent applications were permitted during the 6-week period of use, for example, if the participant washed their hair more regularly. The placebo comparator was superficially identical and applied in the same way and at the same rate. Both required shaking before application and had a warning to avoid spraying onto the face to prevent eye irritation.

At enrolment and at cross-over between using the different treatment sprays, we provided treatment to all participants to eliminate any lice already present, even if none were detected. For this we used dimeticone $4 \%$ liquid gel (Hedrin Once liquid gel, Thornton \& Ross, UK) applied for $15 \mathrm{~min}$ before washing, with a repeat treatment after 7 days, which was not strictly necessary due to the high level of efficacy exhibited by the product, ${ }^{9}$ but the second application was a requirement for approval of the study by the Medicines and Healthcare Products Regulatory Agency (MHRA) assessor. We used the same product to treat infestations that participants and household members acquired during the course of the study. Participants who were found to have contracted an infestation at any point were not withdrawn. Treatments were applied by investigators. Participants who had been infested continued to use their designated spray during the period between applications of dimeticone liquid gel because this therapeutic product is non-residual and thus conferred no protective effect between treatments. 
At the beginning of the study an instruction sheet was supplied to the parent/carer(s) for use of the sprays. At weekly intervals an investigator visited each family to check the participant for lice using a detection comb, supply a new bottle of spray and return the used bottle to the study centre for weighing to determine the quantity used.

\section{Definition of infestation}

We expected that some lice would be found while using the preventive spray because it was possible that participants may have picked up lice at school during the afternoon prior to the visit. Therefore, no action was taken on first finding of lice unless there were five or more large lice (adult and third stage nymphs) or if there were any small nymphs (first and second stage nymphs) present. Either of these was evidence that an infestation had been present for some time. Young nymphs would only be present if eggs had been laid on the head and most reinfestation events start with fewer than five adult or third stage nymphal lice. If lice of any stage were discovered on two consecutive visits, this was considered primary evidence of an ongoing infestation. When infestation was confirmed it was treated using two applications a week apart of dimeticone $4 \%$ liquid gel, and any lice discovered were fixed into the case record using clear tape as confirmation.

At the end of each of the 12 weeks of follow-up, the assessor noted whether:

1. There were any live lice present.

2. Lice were found at previous assessment but no action was taken.

3. There were more than five lice.

4. Any stage 1 or 2 nymphs were present.

If an assessor found there were any live lice present (' 1 ') and, if at the same time, any of '2', ' 3 ' or ' 4 ' also applied, this was considered to be an active infestation. The lice were collected and fixed into the case record book, and the participant treated to eliminate infestation. The numbers of each development stage, and the total numbers of lice were recorded after examination in the laboratory.

\section{Objectives}

The study objective was to demonstrate that with regular use, $1 \%$ octanediol spray could protect against head lice establishing an infestation by killing any lice that crawled onto the treated hair. Unlike a repellent, we recognised that lice would not be inhibited from crawling onto the head but that the product should, if it was applied correctly, be effective in limiting the risk of an infestation becoming established for people using it.

\section{Outcomes}

The primary outcome measure was the time to first infestation, identified using systematic detection combing over the whole head. Secondary endpoints were whether infestations occurred at any time while using the product and the safety of the spray in use.

\section{Sample size}

This study was designed to detect the superiority of $1 \%$ octanediol product compared with a placebo. The study was of an unusual type for clinical investigations because, unlike most clinical investigations, the participants in this study did not already have a treatable condition. The aim to prevent a treatable condition was also unlike other 'preventive' studies, for example, vaccine trials, in that those are normally long-term population studies engaging large numbers of participants with a quite small potential for detectable failure overall.

We proposed a cross-over design because it allowed smaller numbers of participants to be involved, also allowing each participant to act as his/her own control. Developing the design was difficult because the risk factors for each individual were unknown, so randomisation alone may not wholly address any disparity in infestation risk due to social and family circumstances, especially in a relatively small study cohort. Consequently, self-controlling for each individual was an attractive option to avoid any skew resulting from these unknown factors.

Primary analysis, based on time-to-onset of first infestation, was considered a more powerful method of detecting differences between $1 \%$ octanediol and placebo than a simpler approach based on whether or not a participant got an infestation.

Sample size calculations by the statistical consultant (see online supplementary file) were based on 10000 simulations of cross-over studies using a range of defined study sizes, setting the power to detect a difference significant at the 95\% CI, and then estimating the minimum sample size to obtain $80 \%$ or $90 \%$ power. For risk of infestation, we looked at the experience of participants in previous studies of between three and five instances of reinfestation per year, estimated to be equivalent to a rate per person per week of about 6 $10 \%$. From this we expected a reduction of risk between $60 \%$ and $70 \%$ when using the active spray. Consequently, we selected a sample size of 68 participants based on assumed weekly infestation rates of $6 \%$ for placebo and $2 \%$ for octanediol, based on the estimated sample size for $80 \%$ power of 64 plus allowance for dropout. These were equivalent to weekly rates of survival from infestation of $94 \%$ for placebo and $98 \%$ for octanediol, or 6-week survival rates of $69 \%$ and $88.6 \%$. This sample size gave expectations of 19.8 possible infestations for placebo and 7.8 for octanediol over the course of the study.

\section{Randomisation: allocation concealment}

The randomised treatment allocation code was generated using the free online randomisation service at http://www.randomization.com/, seed number 26438 created on 10 October 2012. Treatment allocation was 
made in eight balanced blocks of 10 treatments, with one spare block randomised in case replacements were required.

The treatment allocations bore the anonymous identification of the product to be used and instructions for application. The product identification/instruction sheets were sealed in opaque sequentially numbered envelopes with the participant number taken from the randomisation schedule. This study operated in a crossover design with each participant acting as their own control so all participants used both preparations during the course of the study. The product codes were not broken until after the completion of data collection, entry of data into the study database and database lock.

\section{Statistical analysis}

Analyses testing for differences between the treatments accounted for the cross-over design and were based on within-participant differences between effectiveness of $1 \%$ octanediol compared with placebo during the respective 6-week treatment periods. Primary data management and analyses were performed by PN Lee Statistics and Computing Ltd in collaboration with the investigators. Binary data were analysed using the McNemar test and counts and ranked data using the Wilcoxon signed-rank test for paired data. We analysed participants overall and separately in each randomisation arm, according to which treatment they received first.

For the primary outcome, the time to the first confirmed infestation, we used a seven-point ranking to score the participants:

$1=$ Infestation first confirmed at the first follow-up assessment

2=Infestation first occurred at the second follow-up assessment

6=Infestation first occurred at the sixth follow-up assessment

$7=$ No infestation confirmed in the six assessments

Other endpoint analyses included whether infestation occurred at any time, how many new infestations occurred during each 6-week treatment period and the number and types of adverse events.

For the primary outcome, we used Kaplan-Meier curves to illustrate the time pattern of survival of the participants from infestation either when using $1 \%$ octanediol or when using placebo. We did not test differences between the treatments for significance using the log-rank test because the two curves were nonindependent being based on the same participants.

We performed analyses on the intention-to-treat (ITT) and per-protocol (PP) groups. Prior to the start, we anticipated some dropouts, mostly during the second 6 -week period of treatment. In order to address this problem, if it arose, we planned analyses to allow for dropouts by making an assumption that this would be due to infestation. Thus for analysis of dropouts we assumed that an infestation had occurred in the first week that a follow-up was not possible. If this were to happen in the first treatment period, so there were no data for the second period, we made the assumption that the same response would have occurred in both 6 -week periods. However, based on previous experience in this community, we also anticipated that dropout, were it to occur, would arise at a very low rate that would not require censoring or other specific measures to address the issue in the analyses.

We also analysed baseline characteristics to compare participants according to which product they were randomised to receive first. These data were compared using Fisher's exact test for binary data and the Mann-Whitney U test for counts and ranked variables.

\section{RESULTS}

\section{Participant flow}

We recruited 64 prospective participants but one of those became lost to follow-up after only one prestudy treatment using dimeticone $4 \%$ liquid gel. As this individual had not entered the investigative treatment phase, we considered that they had not actually started participation and should be eliminated from the analyses, leaving 63 enrolled participants in the ITT population, 34 given octanediol followed by placebo and 29 given placebo followed by octanediol. All participants were recruited from the area around Cambridge, UK. The majority were recruited between 22 October and 16 November 2012. All participants had completed both arms of the study by mid-March 2013. Of those recruited, two participants failed to complete the study, one dropped out and one was lost to follow-up.

Twenty participants were so inconsistent in product use that we excluded them from the PP population for protocol deviations. Reasons for exclusion could be classified into five types summarised in figure 1: six participants accidentally used seven bottles of the first study treatment and five bottles of the second study treatment, instead of six bottles for each group; two were given rescue treatments at the wrong time; one was lost to follow-up and one dropped out; two people could not be assessed within the agreed time window on three occasions. Some of these were also found to be in a group that did not apply the products on a regular basis. Altogether 16 participants failed to apply the treatments correctly within agreed protocol limits. Fourteen of these (six when using octanediol and eight when using placebo) failed to use any spray during one or more weeks. Where the spray was only applied once in a given week, when it should have been applied at least twice, it was considered a minor protocol deviation. However, repeated inconsistency in use was considered a major deviation so, for the analyses, two people were excluded from the PP population because they applied the spray only once during a week on three or more occasions.

These participants were included in the ITT analyses but were excluded from the PP analysis. 
Figure 1 Flow chart of participants through the study.

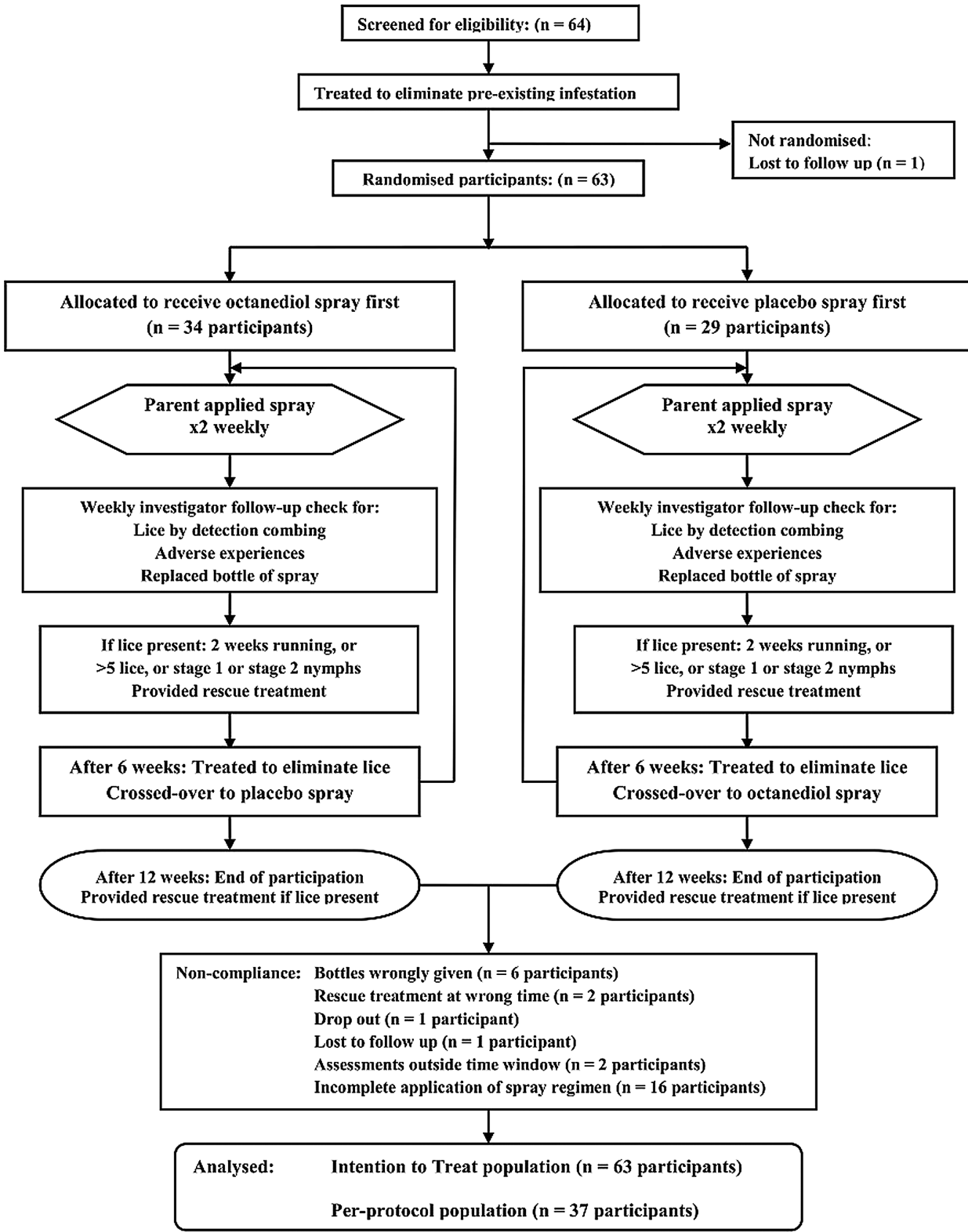

\section{Baseline data}

Of the 63 participants in the investigation phase, 50 $(79.4 \%)$ were girls and $18(28.6 \%)$ were aged 10 years or over, with the remainder aged 4-9 years. There was no significant difference between randomisation groups in age and sex and no significant difference in household size, number of members checked for lice in the household or numbers of people found to have lice at baseline (table 1). Of the household members diagnosed with lice but not enrolled in the study, only one declined treatment to eliminate lice. Similarly, there were no differences between randomisation groups in hair length, degree of curl or hair type. However, there was a significant $(p<0.05)$ difference in hair thickness, with participants allocated octanediol followed by placebo having thicker hair $(52.9 \%$ thick, $32.4 \%$ medium, $14.7 \%$ fine) than those allocated placebo followed by octanediol (24.1\% thick, $41.4 \%$ medium, $34.5 \%$ fine) but, as this was only one of a wide range of variables studied, it was not inconsistent with chance. Fourteen $(22.2 \%)$ participants stated they averaged fewer than two hair washes per week. The percentage was higher for participants allocated placebo followed by octanediol $(34.5 \%)$ than for octanediol followed by placebo $(11.8 \%)$. This difference was nearly significant $(0.05<p<0.1)$. Five $(7.9 \%)$ of the participants dyed their hair, with no difference seen between the randomisation groups. At enrolment, before treatment to eliminate lice, existing infestation was reported as 'None' in 21 $(33.3 \%)$, 'Light' in 20 (31.7\%), 'Moderate' in 12 $(19.0 \%)$ and 'Heavy' in $10(15.9 \%)$. There were no significant differences between randomisation groups and analyses did not suggest any major failure of randomisation. 
Table 1 Demographic characteristics of the study population measured at baseline

\begin{tabular}{|c|c|c|c|c|}
\hline Statistic & $\begin{array}{l}\text { Octanediol } \\
\text { then placebo }\end{array}$ & $\begin{array}{l}\text { Placebo then } \\
\text { octanediol }\end{array}$ & Total & p Value \\
\hline Number of participants & 34 & 29 & 63 & \\
\hline Mean age (years) & 8.12 & 7.72 & 7.94 & NS \\
\hline Percentage of age 1-9 & 70.59 & 72.41 & 71.43 & \\
\hline Percentage of males & 20.59 & 20.69 & 20.63 & NS \\
\hline Mean number living in household & 4.44 & 4.69 & 4.56 & NS \\
\hline Mean number checked for lice & 2.50 & 2.66 & 2.57 & NS \\
\hline Mean number with lice in household & 1.38 & 1.14 & 1.27 & NS \\
\hline Hair length score* & 3.38 & 3.34 & 3.37 & NS \\
\hline Percentage with hair below shoulders & 61.76 & 55.17 & 58.73 & \\
\hline Hair thickness score $†$ & 2.38 & 1.90 & 2.16 & $<0.05$ \\
\hline Percentage with hair thick & 52.94 & 24.14 & 39.68 & \\
\hline Degree of curl score $\ddagger$ & 1.62 & 1.34 & 1.49 & NS \\
\hline Percentage with straight hair & 58.82 & 75.86 & 66.67 & \\
\hline Mean hair type score§ & 1.97 & 2.10 & 2.03 & NS \\
\hline Percentage with hair normal & 97.06 & 89.66 & 93.65 & \\
\hline $\begin{array}{l}\text { Percentage with 'continuous' or 'constant' head lice, } \\
\text { or with }>10 \text { infestations in the last year }\end{array}$ & 35.29 & 44.83 & 39.68 & NS \\
\hline Percentage washing hair less than twice per week & 11.76 & 34.48 & 22.22 & $<0.1$ \\
\hline Percentage using hair dye & 5.88 & 10.34 & 7.94 & NS \\
\hline Mean infestation levelף & 1.29 & 1.03 & 1.17 & NS \\
\hline
\end{tabular}

\section{Outcomes}

All participants indicated that they were at risk of infestation and $42(66.7 \%)$ had an existing infestation at the time of first examination. The remainder stated that they had recently or regularly experienced infestation and anticipated that they were likely to be exposed to further infestation. Of the 63 participants in the ITT population, all but two completed the study. One dropped out and the other was lost to follow-up.

More lice were found during every week when placebo was used compared with the number found when using octanediol (table 2 and figure 2). However, this difference was only found to be significant $(p<0.05)$ for the mean number of stage 2 nymphs at weeks 1 and 6 and almost significant $(0.05<\mathrm{p}<0.1)$ for the mean number of stage 1 nymphs at weeks 4 and 6 .

\section{ITT population}

We found a total of 32 confirmed infestations in 20 participants, which broke down as 12 people (19\%) infested when using octanediol and 17 people (27\%) when using placebo; 10 people using placebo caught lice on two or more occasions (table 2 and figure 2). Infestations occurred in three participants when using octanediol but not placebo, in eight participants when using placebo but not octanediol and in nine participants when using both. In this group, the infestation occurred earlier with placebo than with octanediol in seven participants, earlier with octanediol than placebo in just one and in another the infestations occurred after the same time interval on both treatments (table 2).

This analysis of primary outcome, based on time to first confirmed infestation, showed a significant advantage $(p=0.0129)$ of $1 \%$ octanediol.

Including those who were only infested while using one treatment, there were 15 participants who did better while using octanediol (ie, either they were only infested while using placebo or else they were infested earlier using placebo) compared with 4 who did better with placebo (table 2). This difference is illustrated in figure 3 , the Kaplan-Meier plot of the proportions surviving free from confirmed infestation by week of participation in the study.

The comparison of rate of confirmed infestations, based on the eight people infested only while using placebo, versus the three infested only while using octanediol, did not show a significant difference $(\mathrm{p}=0.2266)$. Overall $19.7 \%$ of participants were found to be infested while using octanediol compared with $27.9 \%$ of those using placebo. Among these we found a significant $(\mathrm{p}=0.0453)$ advantage to $1 \%$ octanediol in relation to the primary outcome of time to first infestation in the group randomised to receive placebo first then octanediol. We found no advantage in the group receiving octanediol followed by placebo.

\section{PP population}

After making allowance for various non-compliance issues, 26 participants were eliminated from the ITT 
Table 2 Numbers of lice recovered from infested participants according to the week of receiving each spray treatment

\begin{tabular}{|c|c|c|c|c|c|c|c|c|c|c|c|c|}
\hline \multirow{3}{*}{$\frac{\text { Treatment }}{\text { Week number }}$} & \multicolumn{12}{|c|}{ Number of lice recovered } \\
\hline & \multicolumn{6}{|c|}{$1 \%$ Octanediol treatment period } & \multicolumn{6}{|c|}{ Placebo treatment period } \\
\hline & 1 & 2 & 3 & 4 & 5 & 6 & 1 & 2 & 3 & 4 & 5 & 6 \\
\hline \multicolumn{13}{|l|}{ Participant } \\
\hline 001 & & & 4 & & & & & 3 & & & & 2 \\
\hline 003 & & & & & & & 3 & & & & & \\
\hline 007 & & & & & & & & 2 & 6 & 5 & & \\
\hline 008 & & & & & & & & & & & & 1 \\
\hline 010 & & & & & & & 3 & & & & & 1 \\
\hline 011 & & & & & & & & & 14 & & & \\
\hline 015 & & & 3 & & & & & & & & & \\
\hline 023 & & & 2 & & & & & & 2 & 4 & & \\
\hline 031 & & & & & & & 3 & & & & & \\
\hline 032 & & & 4 & & & & & & & & & 39 \\
\hline 033 & & & & & & 4 & & & & & 3 & \\
\hline 037 & & & & & 5 & & 3 & & 5 & & & \\
\hline 043 & & & & & & & & & & 2 & 1 & 10 \\
\hline 045 & & & & & 1 & & & & & & & \\
\hline 046 & & & & & & 4 & & & & & & \\
\hline 049 & & & & & & 5 & & & & & 9 & 5 \\
\hline 051 & & & & & 12 & & & & & 1 & 3 & \\
\hline 054 & & & & & & & & & 4 & 4 & 4 & 5 \\
\hline 055 & & & & & & 1 & 1 & & & & & \\
\hline 061 & & & & & & 1 & & & 3 & 2 & & \\
\hline Number of infestations & 0 & 0 & 4 & 0 & 3 & 5 & 5 & 2 & 6 & 6 & 5 & 7 \\
\hline Cumulative number of infestations & 0 & 0 & 4 & 4 & 7 & 12 & 5 & 7 & 13 & 19 & 24 & 31 \\
\hline Total lice & 0 & 0 & 13 & 0 & 18 & 15 & 13 & 5 & 34 & 18 & 20 & 63 \\
\hline
\end{tabular}

population to leave 37 in the PP analysis. Twenty-three of these were randomised to receive octanediol first. No significant differences were seen between treatments for any of the three outcomes considered, even at $\mathrm{p}<0.1$. However, the pattern was similar to that seen in the ITT population, with:

Intention to treat population

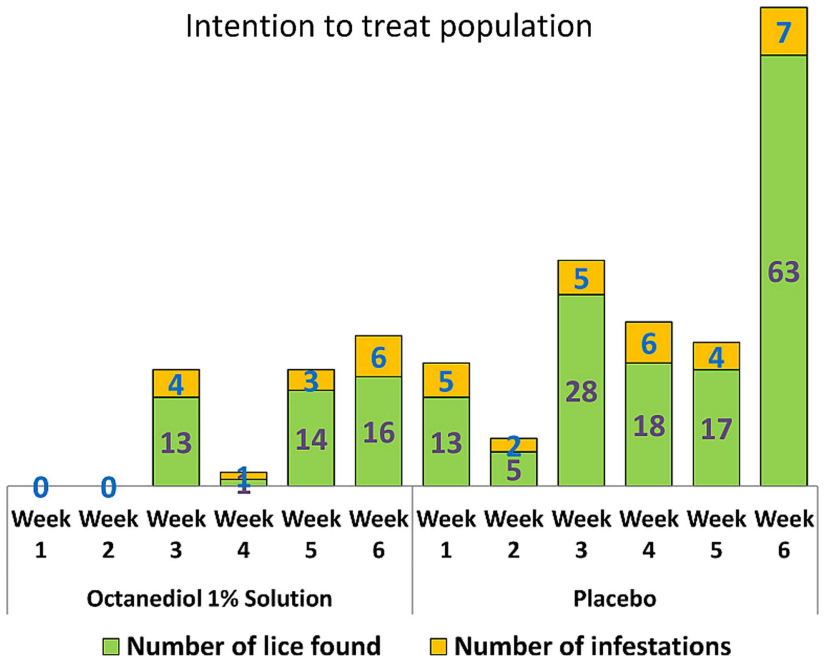

Figure 2 Relative number of infestations and numbers of lice recovered between the two treatments in the intention-to-treat population.
A higher frequency of confirmed infestations using placebo $(24.3 \%)$ than with octanediol (16.2\%).

- A shorter time to first infestation, with the mean scores 6.11 for placebo and 6.62 for octanediol.

The Kaplan-Meier comparison plot is shown in figure 4.

Analyses of the rates of infestation, taking into account the various demographic characteristics, showed no significant difference between the two treatments.

\section{Product use}

Measurements of spray use were based on the bottle weights. The average use per bottle was $17.35 \mathrm{~mL}$ for octanediol spray and $18.9 \mathrm{~mL}$ for placebo. For octanediol average usage varied from 2.33 to $62.08 \mathrm{~mL}$ and for placebo from 1.43 to $66.32 \mathrm{~mL}$ in each week. These quantities were partly influenced by the number of applications, with a few participants applying the spray daily. However, the quantity used per application apparently varied greatly and several people were less than accurate in the information they provided, either in the reported number of spray applications or in a few cases whether they had used the bottle at all.

\section{Adverse events}

There were no serious adverse events and no adverse events that were considered probably related to treatment. The majority of adverse experiences were 


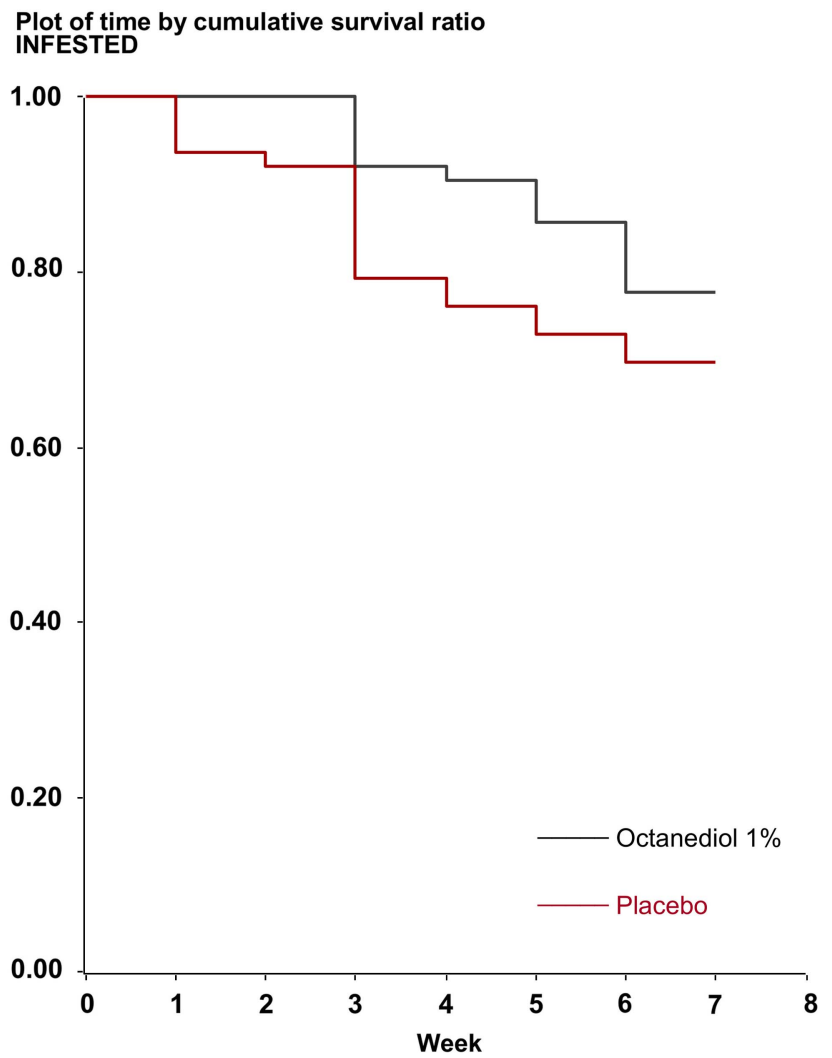

Figure 3 Kaplan-Meier plot of time to infestation intention-to-treat population.

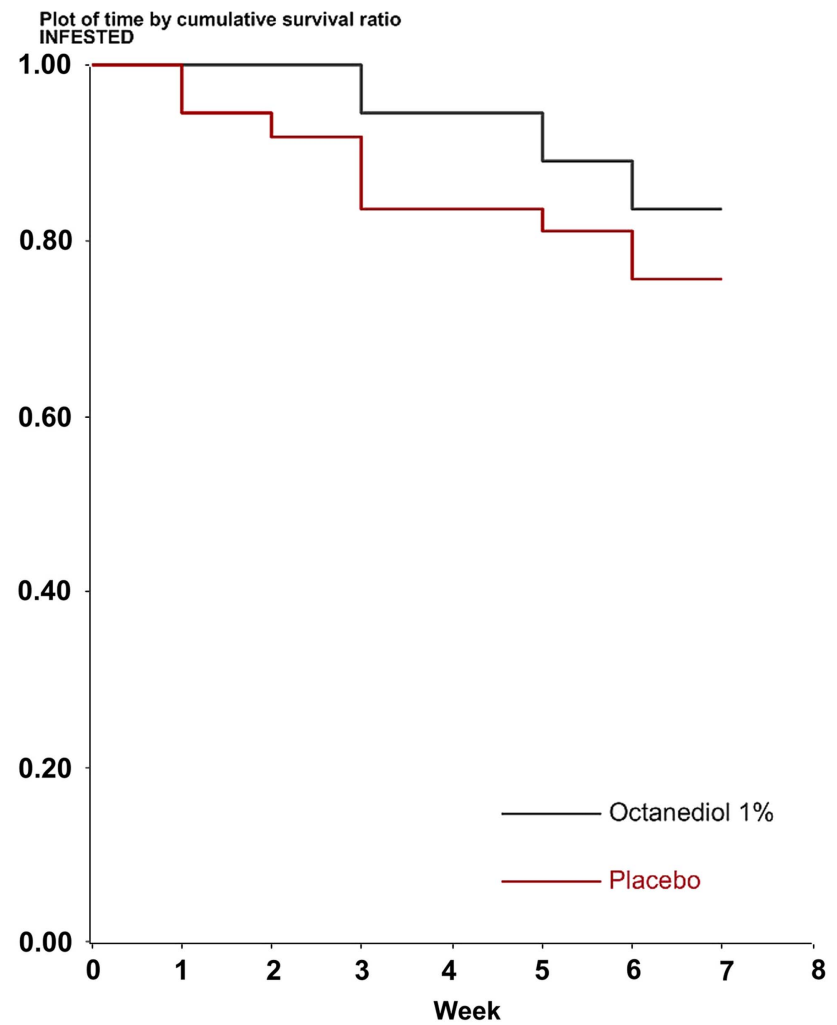

Figure 4 Kaplan-Meier plot of time to infestation per-protocol population. common childhood ailments and minor accidents to be expected in any population of this age range over a moderately prolonged observation period. There were two adverse events considered possibly related to treatment while using $1 \%$ octanediol. The first, a rash of moderate severity, required concomitant medication and was resolved in 5 days. The other, application site erythema, was mild, required no action and resolved the same day.

\section{DISCUSSION}

We have conducted the first investigation of a nonrepellent product intended to prevent head lice from establishing an infestation. We found that with regular use there was a significant $(\mathrm{p}=0.0129)$ difference in time to first infestation when using $1 \%$ 1,2-octanediol spray compared with placebo. There were also non-significant trends for a reduced risk of contracting an infestation and for lower numbers of lice surviving if users did become infested.

There are no data on incidence of head louse infestation from any source yet. Prior to the start, we needed to make estimates of the number of infestation exposures likely to occur during the study period. We made an estimate of the underlying weekly probabilities of infestation in school children based on sales of pediculicides, adjusted for age group at risk, repetition of treatment, overall population and local population sizes. The indicated risk, based on the school-aged population in general, suggested we could expect an infestation rate in the study group of approximately 0.31 cases/week, meaning for a 12-week study we could expect only around four cases to arise. Such a rate was clearly unsatisfactory and would not allow us to detect a difference between the two treatments. However, because we planned to primarily recruit from a population known to have experienced repeated infestations, and using data relating to when those people had contracted lice after study treatments, we found we could expect a risk of about 3-5 infestations/year/individual, that is, a risk of about 3.6-4 possible reinfestation contacts per week for the whole group. However, we could not predict how many of these contacts would result in infestations. In practice we could not measure the number of 'possible' infestation events, although we did observe and treat infestations in relatives throughout the study. The result was 32 confirmed infestations, an average of 2.67 each week, in addition to observed lice that failed to establish an infestation, close to our risk estimate.

We expected some infestations either because people did not apply sufficient octanediol or else because it was applied inconsistently. It was also possible that more than five lice could transfer at one time, so if they were seen before the treatment had taken effect it could be mistakenly diagnosed as an infestation. This was most likely in participants with siblings contracting lice regularly, such as those participants who acquired infestations 
when using the octanediol spray as well as the placebo. Consequently, the primary endpoint was determined as the time to first infestation rather than whether an infestation occurred at all and meant that clear analyses could only be performed on that smaller group of participants experiencing infestations in both arms of the study. Despite this limitation on numbers, the outcomes provided a clear distinction between the treatments with a high level of significance $(\mathrm{p}=0.0129)$.

Unlike repellents, $1 \%$ 1,2-octanediol is non-volatile but we do not know how effective it remains between hair washes, which is why the study required a minimum of two equally spaced applications each week. Octanediol is partially water soluble and certainly surfactant soluble at the dose rates applied, so shampooing would remove it, hence regular reapplication was necessary to maintain the protective effect. Our results show this regimen is effective, and would probably have been more effective if participants had applied more product more consistently throughout the treatment period. In this respect, more thorough (or more frequent) applications may be appropriate at times of outbreaks of infestation in the local or school communities.

Many families have long wished for a preventive preparation. They may monitor and treat their own children but these efforts have been undermined by friends and neighbours who are less assiduous in their efforts or do not attempt to eliminate lice at all. We have found that $1 \%$ octanediol spray can prevent lice from establishing and delay onset of infestation when exposure is common. However, although all the carers professed to be concerned about lice, the level of inconsistency of use suggests that relatively few will truly benefit from such a product unless they are prepared to invest the effort to use it properly. Nevertheless, if a high proportion of households in a community were to use a preventive it is possible that the background level of infestation could be reduced to the point where transmission becomes rare compared with when controlled by therapeutic agents alone. One approach to answering this question would be to conduct a study in which a whole community is provided with the protection spray, rather than relatively isolated individuals, over a period of some months and the impact on infestation evaluated.

Acknowledgements The authors would like to thank Dieno George, Steve Skilleter, Ashley Brierley and Nigel Cooper for logistical and technical support for and provision of study supplies. Investigation team members who contributed to the studies but do not meet criteria as authors include Audrey Pepperman, Georgina Baldwin and Dr Paul Silverston, who acted as clinical contact and adverse event reviewer. Monitoring of documentation for completeness and compliance with GCP was by Janet Selby-Sievewright of SynteractHCR, Inc, acting on behalf of the sponsor.

Collaborators Peter N Lee.

Contributors IFB and ERB were responsible for conception and design of the study. All authors were involved in data collection and management. IFB performed some of the statistical analyses and was responsible for drafting and revising the manuscript. All authors approved the final version of the manuscript. IFB is the guarantor.

Funding Thornton \& Ross Ltd funded this trial.

\section{Competing interests None.}

Ethics approval Ethical approval for this study was granted by the National Research Ethics Service Committee North East-Northern and Yorkshire, Reference 12/NE/0253.

Provenance and peer review Not commissioned; externally peer reviewed.

Data sharing statement Participant level data are available from the corresponding author at ian@insectresearch.com.

Open Access This is an Open Access article distributed in accordance with the Creative Commons Attribution Non Commercial (CC BY-NC 3.0) license, which permits others to distribute, remix, adapt, build upon this work noncommercially, and license their derivative works on different terms, provided the original work is properly cited and the use is non-commercial. See: http:// creativecommons.org/licenses/by-nc/3.0/

\section{REFERENCES}

1. Peock S, Maunder JW. Arena tests with piperonal, a new head louse repellent. J $R$ Soc Health 1993;113:292-4.

2. Mumcuoglu KY, Galun R, Bach U, et al. Repellency of essential oils and their components to the human body louse, Pediculus humanus humanus. Entomol Exp Appl 1996;78:309-14.

3. Maunder JW. Novel phenomena arising from the use of acetylcholineesterase inhibiting insecticides against human head lice. Clin Exp Dermatol 1981;3:31-7.

4. Burgess I. Malathion lotions for head lice-a less reliable treatment than commonly believed. Pharm J 1991;247:630-2.

5. Burgess I, Veal L, Sindle T. The efficacy of $d$-phenothrin and permethrin formulations against head lice: a comparison. Pharm $J$ 1992;249:692-3.

6. Burgess IF, Brown CM. Management of insecticide resistance in head lice, Pediculus capitis (Anoplura: Pediculidae). In: Robinson WH, Rettich FRambo GW, eds. Proceedings of the 3rd International Conference on Control of Urban Pests. Prague: Grafické závody, 1999:249-53.

7. Burgess IF. Dermatopharmacology of antiparasitics and insect repellents. In: Gabard B, Elsner P, Surber C, Treffel P, eds. Dermatopharmacology of topical preparations. Heidelberg: Springer-Verlag, 1999:157-78.

8. Burgess IF, Lee PN, Kay K, et al. 1,2-Octanediol, a novel surfactant, for treating head louse infestation: identification of activity, formulation, and randomised, controlled trials. PLOS ONE 2012;7: e35419.

9. Burgess IF, Burgess NA. Dimeticone $4 \%$ liquid gel found to kill all lice and eggs with a single 15 minute application. BMC Res Notes $2011 ; 4: 15$ 